\title{
Displaced Heavy Neutrinos at the LHC and Beyond
}

\author{
Wei Liu* \\ University College London, \\ Gower Street, London WC1E 6BT, UK \\ E-mail: wei.liu.16@ucl.ac.uk
}

\begin{abstract}
We investigate the pair-production of right-handed neutrinos via the Standard Model (SM) Higgs boson in a gauged $B-L$ model. The right-handed neutrinos with a mass of few tens of $\mathrm{GeV}$ generating viable light neutrino masses via the seesaw mechanism naturally exhibit displaced vertices and distinctive signatures at the LHC. We focus on the displaced leptonic final states arising from decays of the SM Higgs, and analyze the sensitivity reach of the LHC and its beyond in probing the active-sterile neutrino mixing. We also analyse pair production of right-handed neutrinos from the decay of the additional neutral gauge boson $Z^{\prime}$. This is interesting especially when $Z^{\prime}$ is relatively light which can lead to displaced vertices in the forward direction. We perform a similar simulation focused on displaced final states at FASER 2, MAPP*, CODEX-b and LHCb and MATHUSLA, CMS detectors for such a process.
\end{abstract}

ALPS 2019 An Alpine LHC Physics Summit

April 22 - 27, 2019

Obergurgl, Austria

${ }^{*}$ Speaker. 


\section{Introduction and Motivation}

The observation of light neutrino masses and mixing from neutrino oscillation experiment indicates the existence of physics beyond the Standard Model (SM). In order to explain the light neutrino masses, a simple ultraviolet complete model based on the gauge group $U(1)_{B-L}$ with three additional right-handed (RH) Majorana neutrinos that can generate neutrino masses via type-I seesaw mechanism is introduced [1]. This model contains a SM singlet Higgs $\chi$ which spontaneously breaks the $B-L$ symmetry giving a Majorana neutrino mass term for the heavy neutrinos $N_{i}$. Besides, there is an additional gauge boson $Z^{\prime}$ which couples to fermions including the RH neutrinos via their $B-L$ charges with the associated gauge coupling $g_{1}^{\prime}$. Thus, heavy neutrinos can be pairproduced in colliders either from the SM Higgs decays via the Higgs mixing $\sin \alpha$, or from $Z^{\prime}$ decays. As the heavy neutrinos can be long-lived when the active-sterile mixing is tiny, they could display signatures of displaced vertices at the LHC as well as in proposed searching for long-lived particles facilities. Particularly, if the $Z^{\prime}$ mass is small, the heavy neutrinos are likely to be produced in the forward direction where the LHCb and proposed detectors such as FASER 2, MAPP* and CODEX-b are placed.

In this short proceeding report, we first introduce the $U(1)_{B-L}$ briefly then we discuss two analyses based on Refs. [2, 3] with displaced vertex signatures of the heavy neutrinos from either the SM Higgs or $Z^{\prime}$ at the LHC and beyond, to probe heavy neutrinos masses and their mixing to the light neutrinos.

\section{2. $B-L$ Model}

The gauge group of the $U(1)_{B-L}$ model is $S U(3)_{c} \times S U(2)_{L} \times U(1)_{Y} \times U(1)_{B-L}$. It contains an Abelian gauge field $B_{\mu}^{\prime}$, a SM singlet scalar field $\chi$ and three right-handed neutrinos $v_{R_{i}}$ to the SM particle content. After spontaneous breaking of the $B-L$ symmetry, we have additional mass eigenstates as $Z^{\prime}, h_{2}$ and $N_{i}$ corresponding to $B_{\mu}^{\prime}, \chi$ and $v_{R_{i}}$. The second Higgs $h_{2}$ is assumed to have a mass larger than the SM Higgs mass $m_{h_{1}} \equiv m_{h_{S M}}$, and it also couple to the SM states via the Higgs mixing $\sin \alpha$ :

$$
\left(\begin{array}{l}
h_{1} \\
h_{2}
\end{array}\right)=\left(\begin{array}{cc}
\cos \alpha & -\sin \alpha \\
\sin \alpha & \cos \alpha
\end{array}\right)\left(\begin{array}{l}
H \\
\chi
\end{array}\right) .
$$

The mass eigenstates of the right-handed neutrinos are called heavy neutrinos, which also mix to the active neutrinos via active-sterile mixings $V_{l N_{i}}$ :

$$
\left(\begin{array}{c}
v_{L} \\
v_{R}
\end{array}\right)=\left(\begin{array}{cc}
V_{L L} & V_{L R} \\
V_{R L} & V_{R R}
\end{array}\right)\left(\begin{array}{c}
\mathrm{v} \\
N
\end{array}\right) .
$$

In the type-I see-saw mechanism, the relation between the two neutrino mass eigenstates $m_{v_{i}} \approx$ $m_{N} V_{l_{i} N}^{2}$ is obtained. In this proceeding, we assume flavour diagonal mixing and focus on the heavy neutrinos coupling to the muons, i.e. $V_{\mu N} \neq 0$. For a detailed description of the model, we refer the reader to Ref. [2,3] and references therein. 


\section{Heavy Neutrino Production and Decay}

Heavy neutrino production via SM gauge bosons has been discussed in numerous studies. Instead, we focus on two additional channels for the production of heavy neutrinos via the SM Higgs and $Z^{\prime}$ as shown in Fig. 1. The SM Higgs is produced via the gluon fusion with Higgs mixing $\cos \alpha$, then it couples to the heavy neutrinos through the Yukawa terms with Higgs mixing $\sin \alpha$. The Drell-Yan process of heavy neutrinos from $Z^{\prime}$ decays is controlled by the mass of $Z^{\prime} m_{Z^{\prime}}$ and its coupling $g_{1}^{\prime}$. We also assume $m_{Z^{\prime}}=3.33 m_{N}$ to make the decay kinematically accessible.
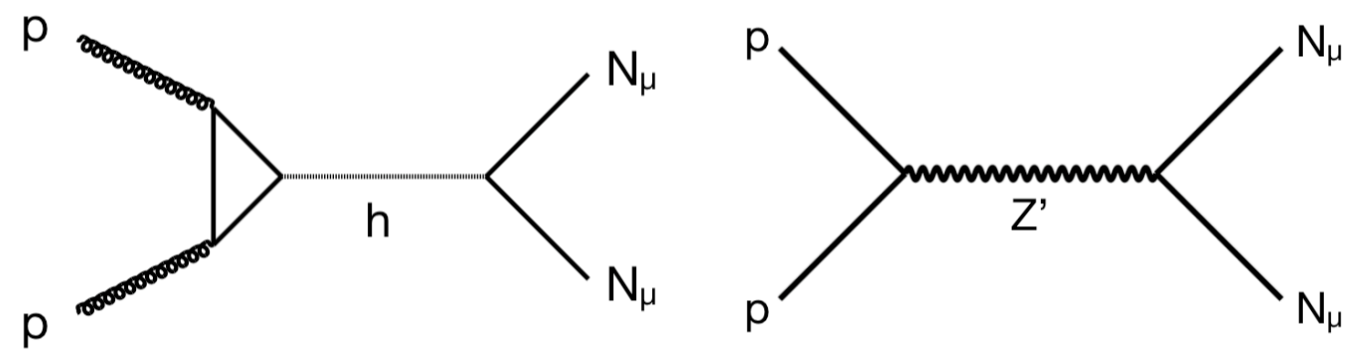

Figure 1: Feynman diagrams of $p p \rightarrow h_{1} \rightarrow N N$ via gluon fusion (left) and $p p \rightarrow Z^{\prime} \rightarrow N N$ (right).

The heavy neutrinos can then decay to SM particles through three-body decays suppressed by the active-sterile mixing. In this proceeding, we focus on the heavy neutrino $N$ which only mixes to the muon neutrino. Thus, for small mixing, the heavy neutrino can be long-lived as the decay length for $m_{N}<m_{Z}$ can be expressed approximately as [2]

$$
L_{N} \approx 0.025 \mathrm{~m} \cdot\left(\frac{10^{-6}}{V_{\mu N}}\right)^{2} \cdot\left(\frac{100 \mathrm{GeV}}{m_{N}}\right)^{5} .
$$

Thus, for $m_{N} \approx 10 \mathrm{GeV}$ with $V_{\mu N} \approx 10^{-5}$, the heavy neutrinos have a decay length of the order of meters.

\section{Displaced Signatures of Heavy Neutrinos at the LHC and Beyond}

The displaced signatures of heavy neutrinos at the LHC and beyond can yield a strong sensitivity on the parameter space $\left(m_{N}, V_{\mu N}\right)$.

For a CMS like detector, we perform such an analysis based on the trigger requirement of Ref. [4] for the final states such as $p p \rightarrow h_{1} \rightarrow N N \rightarrow N \mu^{+} \mu^{-} \nu_{\mu}$,

$$
\begin{gathered}
p_{T}\left(\mu_{1}\right)>26 \mathrm{GeV}, \quad p_{T}\left(\mu_{2}\right)>5 \mathrm{GeV}, \\
|\eta|<2.0, \Delta R>0.2, \quad \cos \theta_{\mu \mu}>-0.75
\end{gathered}
$$

Here $p_{T}\left(\mu_{1,2}\right)$ is the transverse momentum of the first and second leading muon, $\eta$ is the pseudorapidity and $\Delta R$ is the isolation of the two tracks. Background due to cosmic ray muons can be rejected efficiently by correlating the corresponding hits with the beam collision time and with the cut on the angle between the muons, $\cos \theta_{\mu \mu}$ [5]. At LHCb, as it is in the forward direction (as well as FASER 2, MAPP* and CODEX-b), we only perform the analysis for heavy neutrinos from 
light $Z^{\prime}$ decays. We employ a softer trigger requirement from the current LHCb displaced vertex searches $[6,7]$,

$$
\begin{aligned}
& N(\mu)=1, N(j)>0, \quad p_{T}(\mu)>12 \mathrm{GeV} \\
& 2<\eta(\mu, \text { jets })<5, \quad M[\mu j j]>4.5 \mathrm{GeV} .
\end{aligned}
$$

As there are various proposals of new detectors to explore the lifetime frontier at the LHC, we also perform an analysis for these detectors including FASER 2, MATHUSLA, MAPP* and CODEX-b. As the displaced vertices at such detectors are considered to be background-free, we thus assume no kinematical cuts.

The events are simulated using MadGraph5aMC@NLO [8], and the decay length of the heavy neutrinos are calculated as from their exponential decay. The estimated event rates at detectors are obtained after passing the geometrical cuts of the corresponding detector as detailed in Ref. [2, 3].

Figure 2 left shows the sensitive regions in the $\left(m_{N}, V_{\mu N}\right)$ parameter space at 95\% C.L. assuming no observation of a single displaced vertex for the $100 \mathrm{fb}^{-1}$ LHC, the $3000 \mathrm{fb}^{-1}$ HL-LHC and the MATHUSLA option at HL-LHC using the $p p \rightarrow h_{1} \rightarrow N N$ channel. We take the maximally allowed value of the Higgs mixing $\sin \alpha=0.3$ [9]. As a result, we are sensitive to the neutrino mixing of order $10^{-7}$ from the current LHC and one magnitude smaller for the HL-LHC and MATHUSLA for a heavy neutrino with a mass approximately $50 \mathrm{GeV}$.

Figure 2 right indicates the sensitivities for the LHCb and proposed FASER 2, MAPP* and CODEX-b detectors as $p p \rightarrow Z^{\prime} \rightarrow N N$ channel can lead to displaced vertex at the forward direction. The luminosities for these detectors can be found in detail at Ref. [3]. We assume the $B-L$ gauge coupling constant to be the biggest allowed value $g_{1}^{\prime}=10^{-3}$ from the Darkcast [10] and CONTUR $[11,12]$ limits and assume $m_{Z}^{\prime}=3.33 \times m_{N}$ to get the largest event rates. Among all the detectors, even if FASER 2 is placed further away from the interaction point (IP) compared to other detectors, it is not competitive as it is close to beam line and has small radius, thus it is only detecting the heavy neutrinos which are much boosted with smaller proper decay length. MATHUSLA on the other hand, can be sensitive to the smallest active-sterile mixing as it is placed at the top of ATLAS with more than 100 meters away from the IP. As a result, we can reach sensitivities of $10^{-7}$ for the active-sterile mixings from MATHUSLA with $m_{N} \approx 30 \mathrm{GeV}$.

For both channels, we can reach the preferred parameter region where the light neutrinos acquire a mass between $10^{-2} \mathrm{eV}$ and $0.3 \mathrm{eV}$ in a canonical seesaw mechanism which is of great interests.

\section{Conclusion}

Neutrino masses can be generated in the spontaneous $B-L$ symmetry breaking of the minimal $B-L$ model discussed here. In this proceeding, we have described two analyses for the pair-production of heavy neutrinos from the decays of either the SM Higgs or the $B-L$ gauge boson $Z^{\prime}$. Using the displaced vertex signatures of the heavy neutrinos at the LHC and beyond, we obtained sensitivities of order $10^{-7}$ for the active-sterile mixing from both channels for heavy neutrino masses of order $10 \mathrm{GeV}$. The proposed lifetime frontier facilities such as FASER 2, MAPP*, CODEX-b and MATHUSLA are most sensitive to small active-sterile mixing. However, these results are built on the assumption that we choose the biggest allowed value for the Higgs 

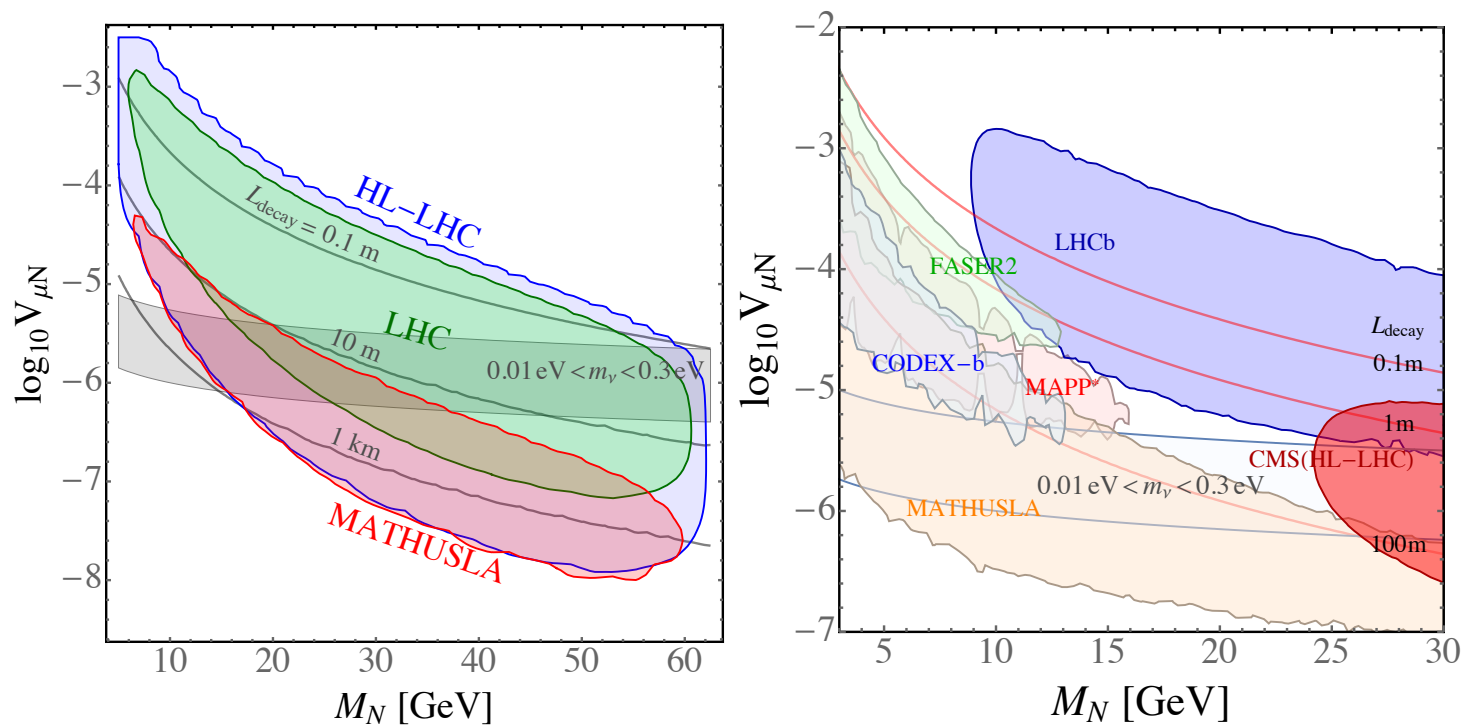

Figure 2: Left: Excluded regions in the $\left(M_{N}, V_{\mu N}\right)$ parameter space at 95\% C.L. assuming no observation of a single displaced vertex for the $100 \mathrm{fb}^{-1}$ LHC (green), the $3000 \mathrm{fb}^{-1}$ HL-LHC (blue) and the MATHUSLA option at HL-LHC (red) from $p p \rightarrow h_{1} \rightarrow N N$ process. Right: Same but for CMS (HL-LHC), LHCb, MATHUSLA, FASER 2, MAPP* and CODEX-b detectors as indicated, at $14 \mathrm{TeV}$ and using luminosity as detailed on Ref. [3] from $p p \rightarrow Z^{\prime} \rightarrow N N$ process. The $U(1)_{B-L}$ gauge coupling and the $Z^{\prime}$ mass are chosen as $g_{1}^{\prime}=10^{-3}$ and $m_{Z^{\prime}}=3.33 \times m_{N}$, respectively. The grey (red) curves denote the proper decay length of the heavy neutrino $N$ and the horizontal band indicates the preferred parameter region where the light neutrinos acquire a mass between $10^{-2} \mathrm{eV}$ and $0.3 \mathrm{eV}$ in a canonical seesaw mechanism. We take an optimistic view assuming no background after selection criteria for both channels.

mixing and $B-L$ couplings as from current constraints. As the limits on these parameters improve, the sensitivity to the neutrino mixing will decrease.

\section{References}

[1] R. N. Mohapatra and R. E. Marshak, Local B-L Symmetry of Electroweak Interactions, Majorana Neutrinos and Neutron Oscillations, Phys. Rev. Lett. 44 (1980) 1316-1319.

[2] F. F. Deppisch, W. Liu and M. Mitra, Long-lived Heavy Neutrinos from Higgs Decays, JHEP $\mathbf{0 8}$ (2018) 181, [1804.04075].

[3] F. Deppisch, S. Kulkarni and W. Liu, Heavy neutrino production via $Z^{\prime}$ at the lifetime frontier, 1905.11889.

[4] E. Accomando, L. Delle Rose, S. Moretti, E. Olaiya and C. H. Shepherd-Themistocleous, Novel SM-like Higgs decay into displaced heavy neutrino pairs in U(1)' models, JHEP 04 (2017) 081, [1612.05977].

[5] CMS collaboration, C. Collaboration, Search for long-lived particles that decay into final states containing two muons, reconstructed using only the CMS muon chambers, .

[6] S. Antusch, E. Cazzato and O. Fischer, Sterile neutrino searches via displaced vertices at LHCb, Phys. Lett. B774 (2017) 114-118, [1706.05990]. 
[7] LHCв collaboration, R. Aaij et al., Search for massive long-lived particles decaying semileptonically in the LHCb detector, Eur. Phys. J. C77 (2017) 224, [1612.00945].

[8] J. Alwall, R. Frederix, S. Frixione, V. Hirschi, F. Maltoni, O. Mattelaer et al., The automated computation of tree-level and next-to-leading order differential cross sections, and their matching to parton shower simulations, JHEP 07 (2014) 079, [1405 . 0301].

[9] A. Ilnicka, T. Robens and T. Stefaniak, Constraining Extended Scalar Sectors at the LHC and beyond, Mod. Phys. Lett. $\mathbf{A 3 3}$ (2018) 1830007, [1803.03594].

[10] P. Ilten, Y. Soreq, M. Williams and W. Xue, Serendipity in dark photon searches, JHEP 06 (2018) 004, [1801.04847].

[11] J. M. Butterworth, D. Grellscheid, M. Krämer, B. Sarrazin and D. Yallup, Constraining new physics with collider measurements of Standard Model signatures, JHEP 03 (2017) 078, [1606 . 05296].

[12] S. Amrith, J. M. Butterworth, F. F. Deppisch, W. Liu, A. Varma and D. Yallup, LHC Constraints on a B-L Gauge Model using Contur, 1811.11452. 